\title{
Ciliary beat frequency in transplanted lungs
}

\author{
D Veale, P N Glasper, A Gascoigne, J H Dark, G J Gibson, P A Corris
}

\begin{abstract}
Background-Patients with lung transplantation are prone to respiratory infections. Generally this is attributable to the effects of immunosuppressive drugs but mucociliary clearance has been found to be impaired in these subjects. A study was performed to determine whether this finding is accompanied by a reduction in ciliary beat frequency (CBF).

Methods-Six patients who had undergone single lung transplantation for fibrosing lung disease were investigated. CBF was measured in mucosal samples from native and transplanted bronchi by a videophotometry method.

Results-The CBF was reduced in the transplanted bronchi in all cases when both fastest and slowest beating cilia were examined. The fastest beating cilia on the native side had a mean (SD) CBF of $12 \cdot 1(1 \cdot 3) \mathrm{Hz}$ compared with $9 \cdot 6(2 \cdot 0)$ Hz on the transplanted side. The slowest beating cilia also had reduced CBF on the transplanted side.

Conclusion-In patients with fibrotic lung disease, CBF is reduced in transplanted bronchi in comparison with native bronchi.
\end{abstract}

(Thorax 1993;48:629-631)

Inhaled particles and endogenous debris are cleared from the tracheobronchial tree by mucociliary transport and cough. ${ }^{1}$ The mucociliary system therefore provides a primary defence mechanism of the respiratory tract. ${ }^{2}$ The system may malfunction either by a primary defect in one of its parts ${ }^{3}$ or as a secondary phenomenon related to infection or exogenous agents. ${ }^{4}$

After lung transplantation patients are prone to pulmonary infection. Their propensity to infection is due not only to immunosuppression but may also be caused by local factors such as reduced cough reflex related to denervation of the airways. Moreover, mucociliary clearance of radiolabelled microspheres is impaired in patients with lung transplants..$^{5-7}$ No differences were found, however, in ciliary beat frequency (CBF) of samples of airway mucosa above and below the anastomosis in a series of patients with heart-lung and single lung transplants. ${ }^{8} \mathrm{We}$ have compared CBF from the airways of normal and transplanted lungs in patients who had undergone single lung transplantation for end stage fibrotic lung disease.

\section{Methods}

PATIENTS

Six patients (four men and two women) of median age 44 (range 22-59) years were studied, all of whom had undergone single left lung transplantation because of respiratory failure related to end stage cryptogenic fibrosing alveolitis.

After lung transplantation the patients were maintained on immunosuppressive treatment with cyclosporin in controlled dose, azathioprine, and prednisolone. They were also taking prophylactic acyclovir. Studies were performed at a median of four months (range eight days to 36 months) after transplantation.

\section{STUDY DESIGN}

Bronchoscopy was performed as part of the regular follow up investigation. The patients had no evidence of infection or rejection at the time of study. The procedure was performed with the subject lying supine after midazolam $5 \mathrm{mg}$ had been given intravenously. No atropine or bronchodilator was used. $\mathrm{SaO}_{2}$ was monitored continuously by finger probe oximetry. Local anaesthetic was applied to the nasal cavity by a cotton bud soaked in $4 \%$ lignocaine which was also sprayed onto the vocal cords. The bronchoscope was passed through the cords and both main bronchi were washed with normal saline.

A nylon sheathed brush was passed through the bronchoscope before any other manoeuvres and the "native" right main bronchus brushed for cilia. The brush was extracted and the tip agitated, suspending the collected mucosa in Medium 199 without Earle's salts (Flow Laboratories Ltd) in a universal container. Mucus samples could then be seen floating in the medium. A new brush was introduced and the procedure repeated on the left (transplanted) side $5 \mathrm{~cm}$ below the suture line. Care was taken to cause only minimal trauma to the bronchial mucosa and to avoid bleeding.

CBF was measured with a method similar to that of Braga et al. ${ }^{9}$ Several drops of the 
suspension were placed in a chamber formed between a microscope slide and a coverslip sealed at the edges with silicone grease. The slide was placed on a heated microscope stage (Micro Instruments, Oxford) and equilibrated to a stage temperature of $45^{\circ} \mathrm{C}$, which previous studies had shown to give a temperature of $37^{\circ} \mathrm{C}$ within the chamber containing the suspension. An image of the mucosal fragments was displayed on a monitor screen using a $\times 200$ oil immersion objective on a microscope fitted with a video camera. The preparation was examined at low power to ensure that samples included at least six ciliated cells. A video recording of 4-10 strips of intact mucosa was made for the later measurement of $\mathrm{CBF}$.

The use of a pinhole collimator and a photodiode probe allowed examination of the beating of two or three adjacent cilia. The probe was placed against the video screen over the image of beating cilia and the movement of the ciliary image was thus recorded as a waveform on the attached oscilloscope. The trace was frozen when a uniform waveform was evident and from this waveform the $\mathrm{CBF}$ was calculated. The use of a video screen allows examination at leisure of the range of $C B F$ across a sample and immediately shows that a range of CBF exists. We have systematically studied this variation in CBF in nasal cilia from normal healthy subjects and shown a coefficient of variation of $9 \cdot 0-27 \cdot 0 \%$ for the fastest beating cilia and of $11 \cdot 1-58 \cdot 0 \%$ for slowest beating cilia. We routinely examine the highest and lowest CBF from all samples and, in addition, two random sites to avoid bias. The CBF was calculated three times at each site on a mucosal sample by rerunning the video and recounting over the same site of the mucosa as defined by a grid system on the screen. Four to ten such samples of mucosa were examined for each subject giving a total number of estimates of CBF of both fastest and slowest beating cilia of 12-30 from each lung sampled.

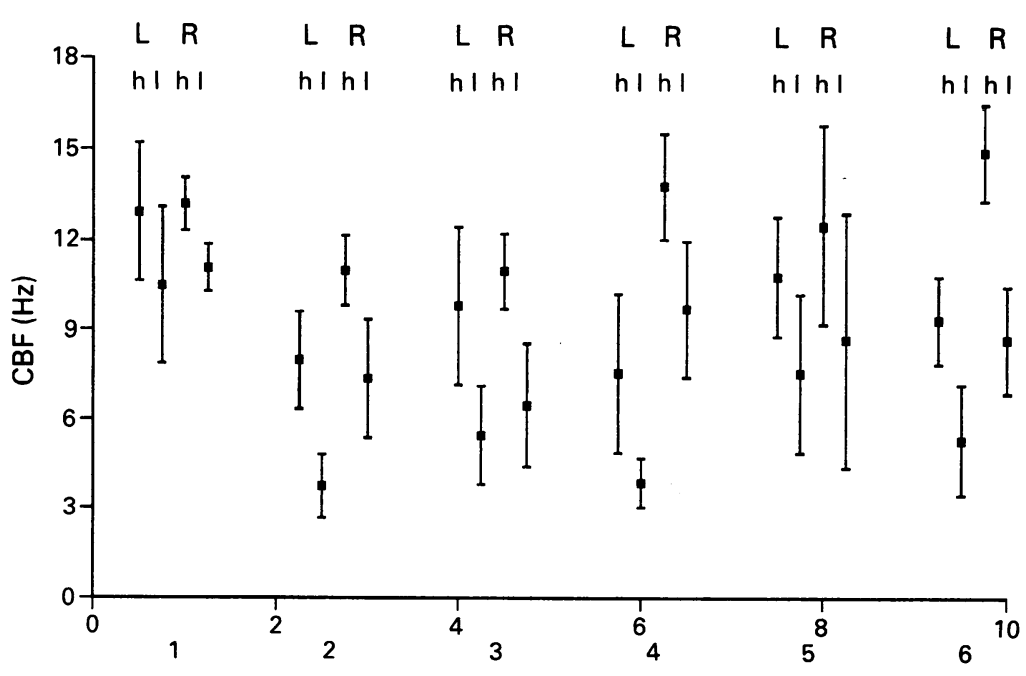

Mean (SD) $C B F$ values for each patient of the highest (h) and lowest (l) readings from the right $(R)$ (normal) and left $(L)$ (transplanted) lung for each individual.
Statistical comparisons were performed with the paired Student's $t$ test.

\section{Results}

The results of CBF from fastest and slowest beating cilia for each lung are shown as mean (SD) of the 12-30 counts (fig). Both the fastest and slowest CBF were reduced in the left (transplanted) bronchus in all cases. The overall mean (SD) CBF of fastest beating cilia from the non-transplanted right main bronchus was 12.1 (1.3) $\mathrm{Hz}$ compared with $9.6(2.0) \mathrm{Hz}$ on the transplanted left side $(p<0.05)$. The corresponding values for the slowest beating cilia were $9.3(0.5) \mathrm{Hz}$ on the right side and $8 \cdot 0(2 \cdot 8) \mathrm{Hz}$ on the left $(\mathrm{p}<0.001)$.

\section{Discussion}

We have shown a reduction in CBF in mucosal samples from transplanted lung when compared with the normal lung in patients who had undergone single lung transplantation. This was consistent in all patients and applied to both the fastest and slowest beating cilia. This finding is consistent with, and may account for, the previously documented reduction in mucociliary clearance from transplanted lungs..$^{5-7}$

The mechanisms of the reduced CBF in transplanted lungs are not clear. Factors which might play a part include the effects of denervation, immunosuppressive drugs, infection, or rejection of the transplanted lung. The possible role of denervation of the airway is unknown, but it has been shown that nasal mucociliary clearance is unaffected by nasal denervation. ${ }^{10}$ Drug effects are unlikely to be relevant as each patient acted as his own control with comparison between transplanted and native cilia. Furthermore, mucociliary clearance is slower in subjects following lung transplantation than in those on a similar immunosuppressive regimen following heart transplantation. ${ }^{7}$

CBF is reduced in the presence of bronchial infection ${ }^{4}$ but the patients studied here had no evidence of infection or rejection in transbronchial biopsy samples taken at the time of ciliary sampling. Reduced CBF might be a result of altered rheological properties of tracheobronchial secretions ${ }^{11}$ in the denervated transplanted bronchus. Mucus secretion is under nervous control ${ }^{12}$ and thus the quantity or composition of mucus may be altered in the transplanted lung. We are unaware of any studies of the properties of mucus secreted by transplanted lungs.

Our results differ from those of Read et al ${ }^{8}$ who showed no reduction in CBF in samples taken proximal and distal to the bronchial or tracheal anastomosis in single lung and heartlung recipients. In their study $\mathrm{CBF}$ was recorded at a single site in 10 mucosal samples whereas we examined the fastest and slowest beating cilia in each sample. There is a wide variability in $\mathrm{CBF}$, even in normal subjects. ${ }^{13}{ }^{14} \mathrm{Six}$ patients in the study by Read 
et al had cystic fibrosis and therefore an effect of persistent infection in the sinuses or airway cannot be excluded. Indeed, two of their patients with cystic fibrosis had a very low CBF proximal to the tracheal anastomosis. It might also be that the heterogeneous nature of their patients may have masked differences between particular groups of patients. We specifically chose a uniform group of subjects, all with lung fibrosis as the underlying disorder. Unlike Read et al we avoided the use of atropine as a premedication because of its effect on mucus secretion which might, in turn, modify CBF.

In conclusion, we have shown that $\mathrm{CBF}$ is reduced in transplanted lung when compared with non-transplanted lung. This may add to the effect of immunosuppression in predisposing to infection in transplanted lungs. Further study is required to elucidate the mechanisms of reduced $\mathrm{CBF}$ in this situation.

1 Pavia D, Bateman JRM, Clarke SW. Deposition and clearance of inhaled particles. Bull Eur Physiopathol Respir 1980;16:335-66.

2 Sleigh MA, Blake JR, Liron N. The propulsion of mucus by cilia. Am Rev Respir Dis 1988;137:726-41.

3 Afzelius BA. The immotile-cilia syndrome and other ciliary diseases. Int Rev Exp Pathol 1979;19:1-43.

4 Wilson R. Secondary ciliary dysfunction. Clin Sci 1988;75:113-20.

5 Dolovich M, Rossman C, Chambers C, Grossman RF, Newhouse M, Maurer JM. Mucociliary function in patients following single-lung or heart-lung transplantation. Am Rev Respir Dis 1987;135:A363.

6 Shanker S, Fulsham L, Read RC, Theodoropoulos S, Cole PJ, Madden B, et al. Mucociliary function after lung transplantation. Transplant Proc 1991;23:1222-3.

7 Silbert D, Cerrina F, Le Roy Ladurie, Chapelier A, Parquin F, Brenot F, et al. Impairment of bronchial mucociliary clearance after lung transplantation. Eur Respir f 1992;5:437S.

8 Read RC, Shankar S, Rutman A, Feldman C, Yacoub M, Cole PJ, et al. Ciliary beat frequency and structure of recipient and donor epithelia following lung transplantation. Eur Respir f 1991;4:796-801.

9 Braga PC, Dall'Oglio G, Bossi R, Allegra L. Simple and precise method for counting ciliary beats directly from the TV monitor screen. $\mathcal{f}$ Pharmacol Methods 1986;16: 161-9.

10 Greenstone $M$. Function and dysfunction of human respiratory cilia: measurement, modification and consequences. MD Thesis, University of Bristol, 1985.

11 Litt M. Mucus rheology: relevance to mucociliary clearance. Arch Intern Med 1970;126:417-23.

12 Seo A. Studies on the nervous regulation of the ciliary movement. Fpn f Med Sci Biol 1931;2:47-75.

13 Yager J, Chen TM, Dulfano MJ. Measurement of frequency of ciliary beats of human respiratory epithelium Chest 1978;73:627-33.

14 Rossman CM, Forrest JB, Lee RMKW, Newhouse MT. The dyskinetic cilia syndrome: ciliary motility in immotile cilia syndrome. Chest 1980;78:580-2. 